\title{
Method and software tool for an optimized passenger orientated connection management
}

\author{
M. Klemenz \& A. Radtke \\ Institute of Transport, Railway Construction and Operation, \\ Leibniz University of Hannover, Germany
}

\begin{abstract}
The trust of the customers in adherence to their expectation to a transport service determines the modal split of the passenger transport. Therefore it has to be a goal for each public transport operation company to maintain and to improve the customer's trust. An important service criteria by a customer accepted public transport offer is the travel time. The travel time depends on the running time, the dwell time, etc., but also on the number of transfer procedures and the transfer waiting time. One of the essential research problems in the surroundings of the connection planning is the question under which condition it is appropriate for the planning of a connection. An important aspect to answer this question is the evaluation of the additional total travel time for all involved passengers in consideration of an achieved (additional travel time for transit passengers in the connecting vehicle) or not achieved (additional travel time for transfer passengers in the feeder vehicle) connection.

The paper develops a new method of an optimized passenger orientated connection management during the planning process (scheduled timetable). The goal is to minimize the additional travel time in consideration of a planned or not planned connection based on the number of all involved passengers. At first it is necessary to identify all influencing variables. After that all boundary conditions will be modelled by using the graph theory. With an adequate shortest path algorithm it is possible to generate connection strategies specific for stations, lines or the whole networks. Particularities of railway operation (e. g. turn around times, operating fleet, timetable interval, conflicts, infrastructure bottlenecks) also can been considered in the model.

For the practical use was developed the software tool ANPLA which is helpful for operating companies or for the timetable construction to solve the set of problems in the surroundings of the connection management. Furthermore the paper includes approaches for a web based application.
\end{abstract}

Keywords: connection management, timetable construction, transit and transfer passenger, graph algorithms, minimized travel time. 


\section{Introduction}

In the surroundings of the connection planning there is one essential research questions:

\section{Under which condition it is appropriate} to plan or not to plan a connection?

An important aspect to answer this question is the evaluation of the additional total travel time for all involved passengers in consideration of an achieved or not achieved connection.

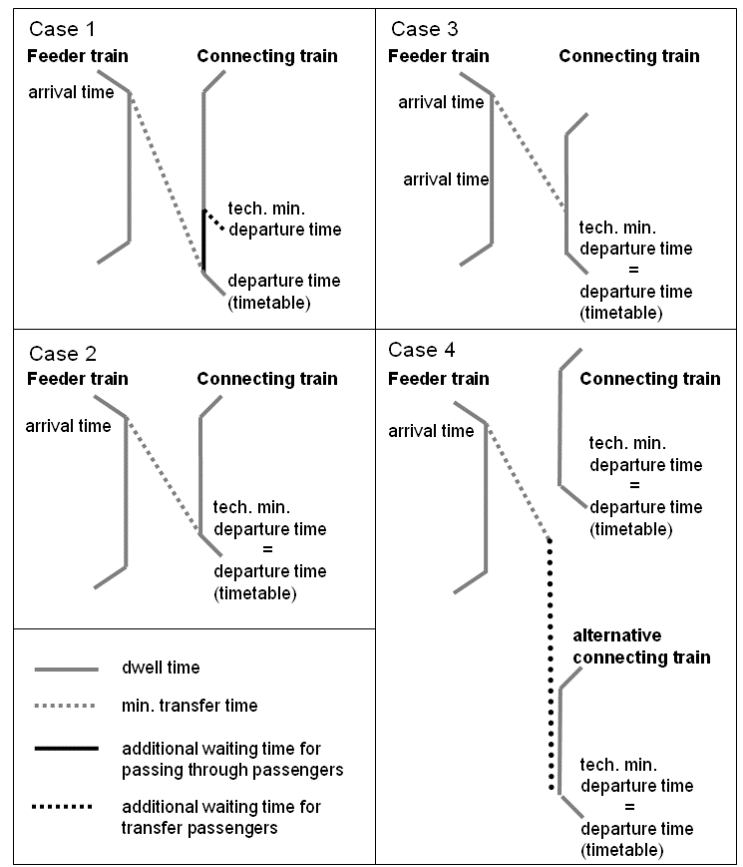

Figure 1: $\quad$ Case study for additional waiting times.

If passengers can reach their final destination only by transferring from one train into another train, the travel time increases according to the transfer time (minimum transfer time and transfer waiting time). The holding of a connection means an extension of the travel time for the passing through passengers (PTP). The no holding of a connection increases the travel time of the transfer passengers (TP). The travel time extension of the PTP in the connecting train and of the TP in the feeder train is normally anti-proportionally. The amount of the additional travel time for all concerned passengers depends of the difference between the arrival time of the feeder train and the departure time of the connecting train (figure 1). Additional dwell times only appear, if the technical minimum departure time of the connecting train is less than the amount of arrival time of the feeder train and the minimum transfer time (cases 1 and 4). 


\section{Influencing factors}

The method for an optimized passenger orientated connection management and the influencing additional total travel depends on several influencing factors:

- Number of transfer passengers

- Number of passing through passengers

- Arrival time of the feeder train

- Minimum transfer time of the feeder train to the connecting train

- Departure time of the connecting train

- Departure time of an alternative connecting train (service interval of the connecting train)

\section{Determination of number of passengers}

The method for an optimized passenger orientated connection management requires information about the modal split, the demand matrix and the time or date of a travel. For the determination of these values different resources are applicable (e.g. statistical evaluation of tickets, interview and count of passengers, positioning with handy ticketing, etc.).

\section{Method for the optimized passenger orientated connection management [7]}

To minimize the additional dwell times in the planning process, it is necessary to develop a method in consideration of the difference between the arrival time of the feeder train and the earliest departure time of the connecting train, required transfer times, the frequency of the connecting train (the earliest departure time of an alternative connecting train) and the number of concerned passengers.

\subsection{Single connection management}

The developed method will be demonstrated at first for a two-train model (figure 2), with transfer passengers from the feeder train to the connecting train (application: connection station is final destination of the feeder train).

Required data input:

- Number of passing through passengers (PTP)

- Number of transfer passengers (TP)

- Number of boarding passengers at connection station, without transfer passengers (BP)

- Number of "on the way" boarding passengers (OBP)

- Distance between the amount of the arrival time of the feeder train and the minimum transfer time and the technical minimum departure time of the connecting train

- Number of "on the way" boarding passengers (OBP) 


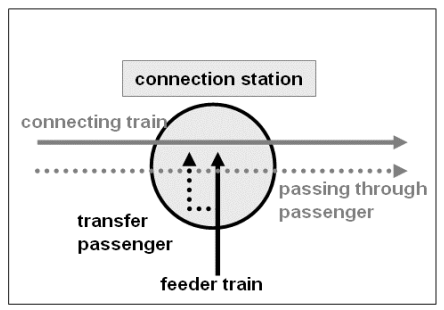

Figure 2: $\quad$ Two train model.

- Distance between the amount of the arrival time of the feeder train and the minimum transfer time and the technical minimum departure time of the connecting train

Technical minimum departure time of an alternative connecting train

This data allows the calculation of the break even point, which defines the time distance between the arrival time of the feeder train (incl. the minimum transfer time) and the departure time of the connecting train. Concerning to a minimized extension of travel times time distance values below the break even point involve a planning of the connection. Time distance values above the break even point entail a no planning of the connection.

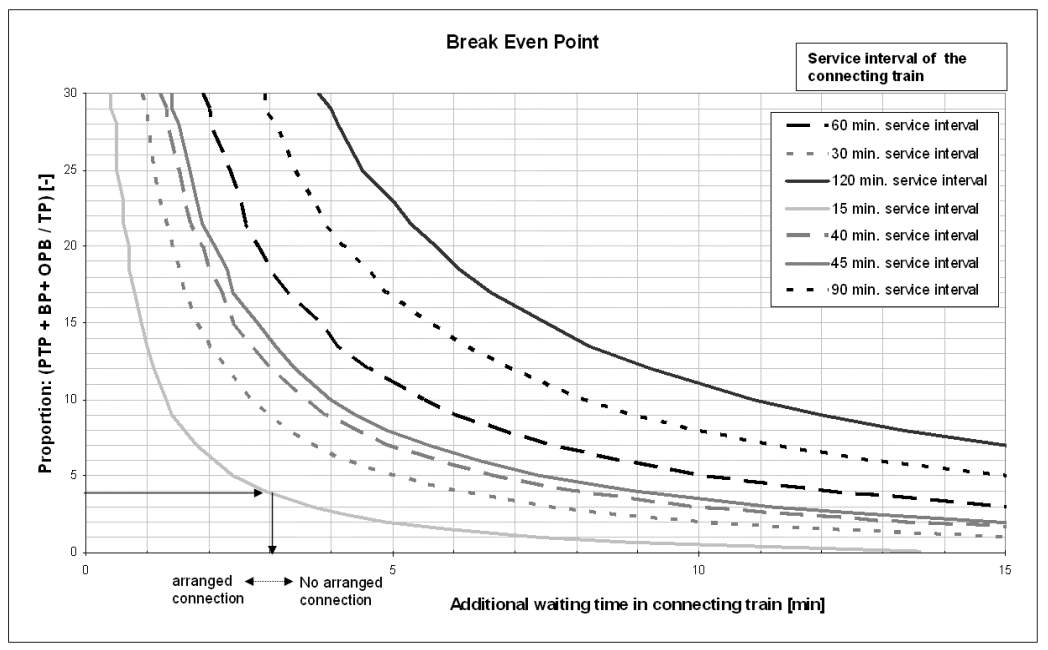

Figure 3: Calculation of the break even point.

The figure 3 shows a abstract of the break even point calculation in correlation of the proportion of the number of transfer passenger and the sum of number of passing through passengers, boarding passengers and on the way boarding passengers and in correlation of the service interval of the connecting train. 
The break even point calculation provides a comprehensive spectrum of applications for the scheduling of timetables:

- Determination of the maximum allowed time distance between the arrival time of the feeder train (incl. minimum transfer time) and departure time of the connecting train.

- Determination of the required service interval of the connecting train in cases of no planed connection strategy.

- Determination of required passing through passengers in cases of a no planed connection strategy.

- Determination of required transfer passengers in cases of a planed connection strategy.

- Determination of required minimum transfer time in cases of a planed connection strategy.

\subsection{Multiple connection management}

In the next step the developed method will be advanced for a station specific $n$ train model (figure 4), with transfer passengers between all involved trains. The model for a station specific connection management depends on graph algorithms. Each graph represents a station with several train connections. The starting node (source) is the pull into a station and the ending node (sink) is the exit of the station. Each involved train has a connection link between source and the second node. This second node represents the arrival time of this train (tan). A waiting link connects the arrival time with a possible departure time (tab) of this train. The number of different waiting time links depends on the number of involved trains. Each waiting time link includes costs and is representing a certain connection strategy of a train. The costs will be calculated based on the additional travel time of all involved passengers. The last link connects the possible departure time with the exit of the station (sink).

To detect an optimal connection strategy it is necessary to compare all the possible waiting links of a train. The link with the lowest costs and therewith the lowest additional waiting time for the concerned passengers is representing the optimal connection strategy. Each train has only one optimal waiting link. The calculation or selection of a waiting link of one train has no influence of the detection of the waiting links of the other trains. This means that the calculation of the optimal connection strategy is for a station specific n-train model for each train independent. The optimal connection strategy for the whole station is the sum of all calculated optimal waiting time links.

To identify the optimal connection strategy it is necessary to calculate the costs of each waiting link $(\mathrm{HK})$ of a train. Therefore two equations $(1+2)$ are necessary:

Equation 1:

Costs of the waiting link $\rightarrow$ Train xo without additional waiting time:

$$
H K_{x o}^{x o}=\sum_{x=x o+1}^{x=n} U_{(x)(x o)} *\left(T_{(x o)}-\left(\tan _{(x)}+t m \ddot{u} z_{(x)(x o)}-t a b_{(x o)}\right)\right)
$$




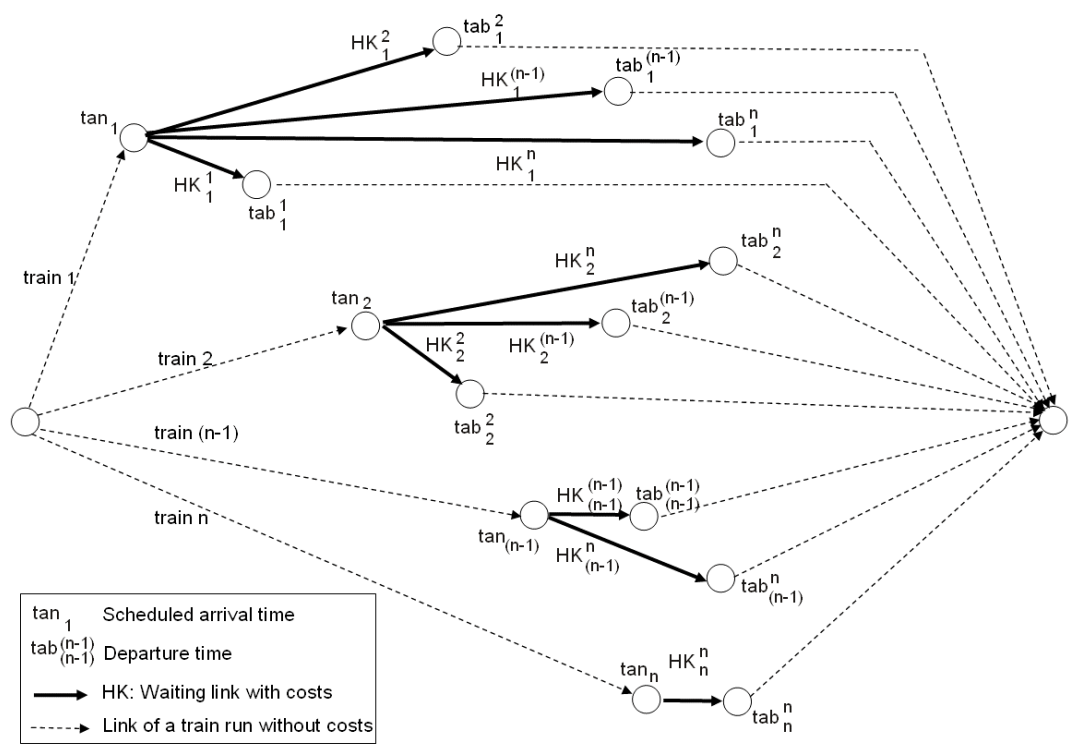

Figure 4: Graph of a station specific n-train model.

with $\mathrm{xo}=1, \ldots,(\mathrm{n}-1)$ and with $\mathrm{x}>\mathrm{xo}$

if $\quad \mathrm{xo}=\mathrm{n} \rightarrow \mathrm{HK}=0$

Equation 2:

Costs of the waiting link $\rightarrow$ Train xo is waiting for train $\mathrm{x}$ $H K_{x o}^{x}=$

(I) $D_{(x o)} *\left(\tan (x)+t m \ddot{u} z_{(x)(x o)}-t a b_{(x o)}\right)+$

(II) $\sum_{y=1}^{y=x o-1} U_{(y)(x o)} *\left(\tan (x)+t m \ddot{u} z_{(x)(x o)}-t a b_{(x o)}\right)+$

(III) $\sum_{z=x o+1}^{z=x-1} U_{(z)(x o)} *\left(\left(\tan _{(x)}+t m \ddot{u} z_{(x)(x o)}\right)-\left(\tan _{(z)}+t m \ddot{u} z_{(z)(x o)}\right)\right)+$

$(I V) \quad \sum_{x=x}^{x=n-1} U_{(x+1)(x o)} *\left(T_{(x o)}-\left(\tan _{(x+1)}+t m \ddot{u} z_{(x+1)(x o)}-t a b_{(x o)}\right)\right)$

with $\mathrm{x}=\mathrm{xo}+1, \ldots, \mathrm{n}$

Description:

(I): Additional travel time for passing through passengers of train xo

(II): Additional travel time for transfer passengers from train $\mathrm{y}$ (which arrives earlier than train xo) into train xo

(III): Additional travel time for transfer passengers from train $\mathrm{z}$ (which arrives later than train xo, but earlier than train $\mathrm{x}$ ) into train xo 
(IV): Additional travel time for transfer passengers from train $\mathrm{x}+1$ (which arrives later than train $\mathrm{x}$ ) into train xo

$\mathrm{U}(\mathrm{x})(\mathrm{xo})$ : Transfer passengers from train $\mathrm{x}$ to train $\mathrm{xo}$

$\mathrm{T}(\mathrm{xo})$ : Departure time of the next connecting train (service interval)

$\tan (\mathrm{x})$ : Arrival time of the feeder train

tab (xo): Departure time of the connecting train

tmüz (x)(xo): Minimum transfer time from train $\mathrm{x}$ to train $\mathrm{xo}$

$\mathrm{D}(\mathrm{xo})$ : Passing through passengers of train xo

$H K_{x o}^{x}$

xo Waiting link: Train xo is waiting for train $\mathrm{x}$

Equation 3:

The link with the minimal costs ( $K \operatorname{Min} Z u g$ xo) is representing the optimal connection strategy per train.

Equation 4:

$$
K_{\text {Min }} Z u g_{x o}=\operatorname{Min}\left\{H K_{x o}^{x o} ; H K_{x o}^{x}\right\}
$$

The sum of all minimal costs $(K$ Min $)$ is representing the station specific optimal connection strategy:

$$
K_{\text {Min }}=\sum_{x o=1}^{x_{0}=n} K_{\text {Min }} Z u g_{x o}
$$

\subsection{Track specific connection management}

In the last step the developed method will be advanced for a n-train model for several stations (figure 5), with transfer passengers between all involved trains. The detection of a optimal connection strategy for several stations is distinctly more complicated and complex than the detection of a station specific optimal connection strategy. The determination of a path with minimal costs can not be done station by station. It must be done simultaneously. The required model also depends on the graph algorithm. To model the connection management for several stations it is useful to use a reference train (yo), which is driving through all involved stations. The graph is representing the whole research area. The source represents the first station of the reference train. The final node represents the destination of the reference train. Links, constrained of the number of possible connection strategies, connect the source with the following nodes. These nodes are representing the new arrival time of the reference train at the following station. The arrival time depends on the chosen connection strategy at the predecessor station. Waiting time links are connecting these nodes with the new arrival times at the next stations. At the last node all possible connection paths are running together. Each waiting link is a possible connection strategy of a train. The costs emerge from the additional travel times of all involved passengers. The detection of the minimum costs overall can not be calculated train by train. Rather the costs have to be calculated for all influenced trains together. Each possible waiting link of a train at a station will be combined with all possible waiting links at the other stations. The link path (per station only one link) with the minimal additional waiting time or travel time for all passengers is 
representing the optimal connection strategy for the reference train and for all influenced trains. For the calculation it will be used the Dijkstra [1-3] algorithm (shortest path or path with minimal costs).

After the detection of an optimal connection strategy follows the verification of the feasibility related to operational aspects. Not conflict free connection strategies must be rejected. Their paths will be blocked and a new calculation will follow.

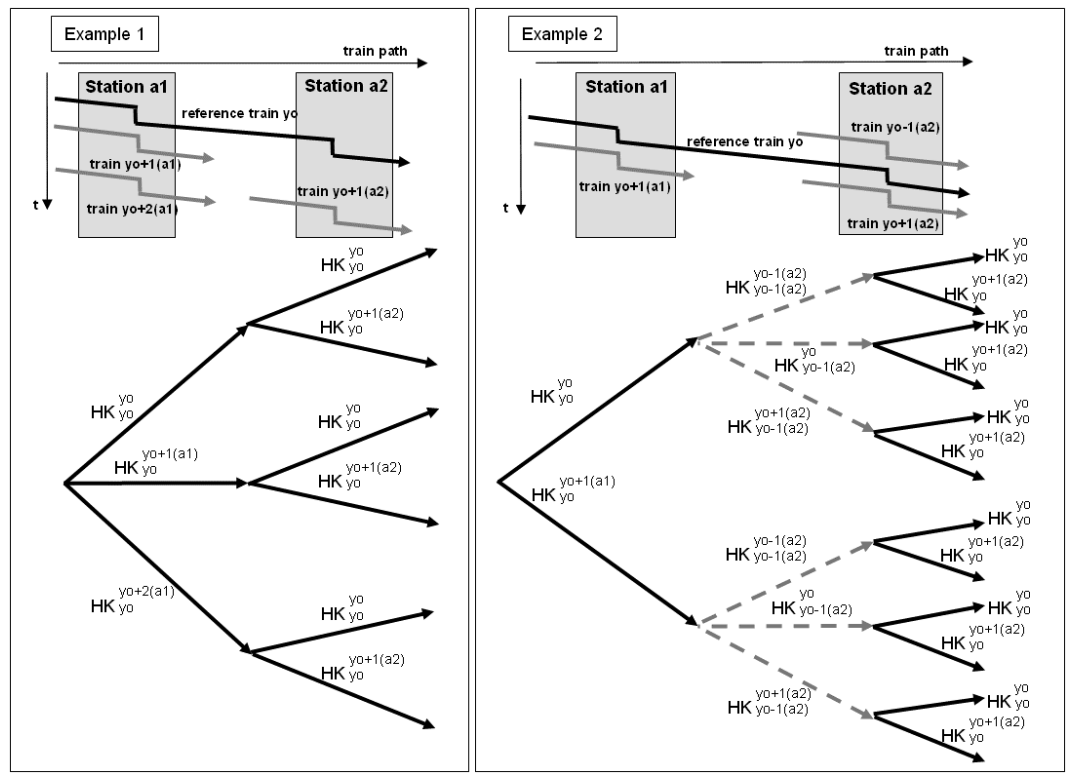

Figure 5: $\quad$ Example for a graph for the n-station and n-train model.

Figure 5 shows two examples of a abstract of a possible graph model. Example one is a very simple graph model. The connection strategies of the reference train yo and the other trains yo $+1(\mathrm{a} 1), y^{+} 2$ (a1) and yo+1 (a2) are independent. They can be calculated by their own.

Example two shows that the connection strategy of train yo is directly connected with the connection strategy of train $y-1(a 2)$. The detection of an optimal connection strategy for the reference train yo is providing at the same time the connection strategy of train yo-1(a2). The connection strategies of other trains yo $+1(\mathrm{a} 1)$ and yo +1 (a2) can be calculated by their own.

The connection strategy of a train, which scheduled departure time at a station occurs earlier than the departure time of the reference train, depends on the chosen connection strategy of the reference train at predecessor stations.

Example 2 in Figure 5 illustrates these dependencies: A reference train (train yo) has fixed arrival times at the stations a1 and a2 depending on the chosen connection strategy at the predecessor station. At station al train 1 has 2 options: It can continue its journey without holding a connection or it can hold a connection to 
train yo $+1(\mathrm{a} 1)$. Therefore the arrival time of train 1 at station a 2 depends on the chosen strategy at station a1. At station a 2 train yo-1(a2) has a scheduled departure time which is located earlier than the possible arrival times of train yo. This means that the waiting time costs of train yo-1(a2) depend on the chosen connection strategy of train yo at station a1. If train yo-1(a2) is not waiting for train yo, the additional travel time of transfer passengers of train yo increase. This additional time is independent of the chosen connection strategy at station a1. If train yo1(a2) is waiting for train yo different additional waiting time costs (depending on the chosen strategy in a1) for the passing through passenger in train yo-1(a2) occur. This waiting time increase depending on postponed arrival time of train yo. To find the minimal additional waiting time for all involved passengers it is necessary to combine all possible connection strategies.

\section{Software tool for the optimized passenger orientated connection management}

For the application of the method of a passenger orientated connection management were developed the software tool ANPLA (figure 6). ANPLA [7] is based on the software and algorithm technology successfully used for the implementation of Dispo $[2,3,6]$, the railway vehicle allocation tool of IVE.

ANPLA calculates for n-trains, n-stations and n- connections depending on the number of concerned passengers, the minimum transfer time, the service interval of the connecting train and the scheduled arrival and departure times the optimized connection strategy of each train. The user has with ANPLA a tool which could answer the question "to plan or not to plan a connection" fast and reliable.

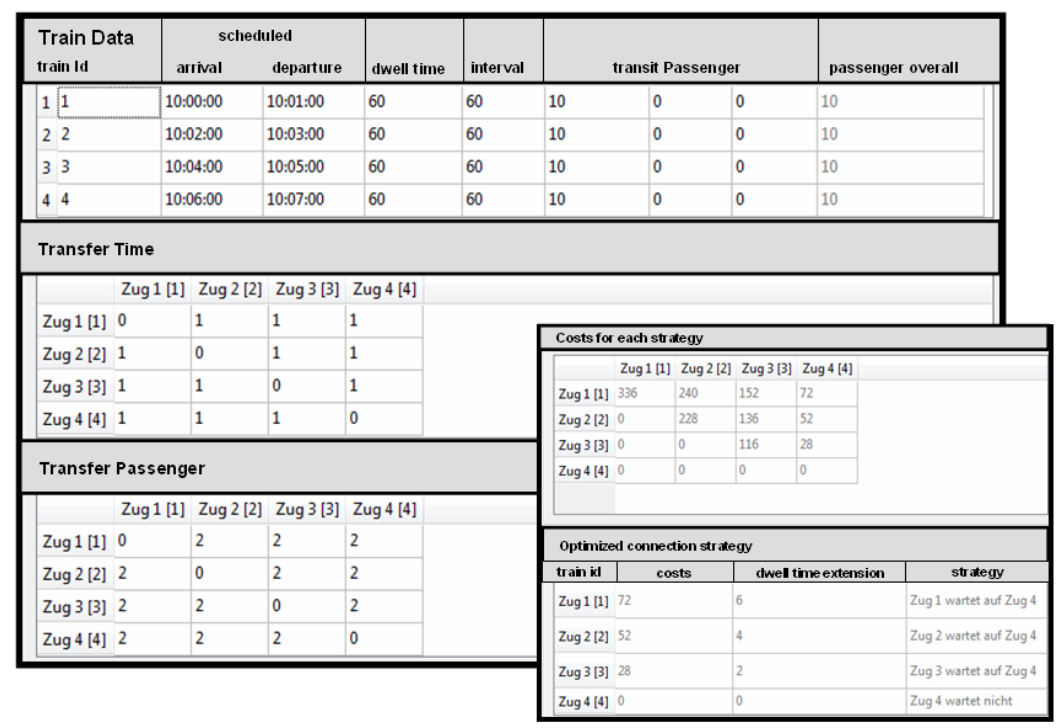

Figure 6: $\quad$ Screenshot: ANPLA. 


\section{Workflow and Web based application}

After the first stand-alone test phase of ANPLA the integration into the RailSysEnterprise environment will be the next step. Figure 7 illustrates a modern workflow between different Train Operating Companies (TOC) and a Railway Infrastructure Undertaking (RIU).

The TOC request train slots and other information from the RIU. In the past, this process in general was a time consuming manual task using telephone, pen and paper or simple spreadsheets. The web based RailSys-CRM (Customer Relation Management) solution for the Hamburg Port Authority (HPA) offers far more possibilities to support this process taking into account the increased time pressure for the planning tasks. TOC and RIU can save time of unnecessary (multiple) data entry and, therefore avoid mistakes. Furthermore, the streaming less flow of data enables the RUI to perform the timetable construction on the bases of the original requests and follow up changes in a much better quality to construct a non-discriminating timetable. Using this technology, the RIU can provide all necessary information to rail regulation authorities to prove the nondiscriminating timetable construction according to the agreed timetable construction rules. Figure 8 shows an example of the web interface of the RailSys-CRM [4, 5] module.

The integration of timetable construction and railway operation simulation enables the RIU to analyse the performance and stability of the timetable before setting the timetable into operation. Analogous to the railway operation

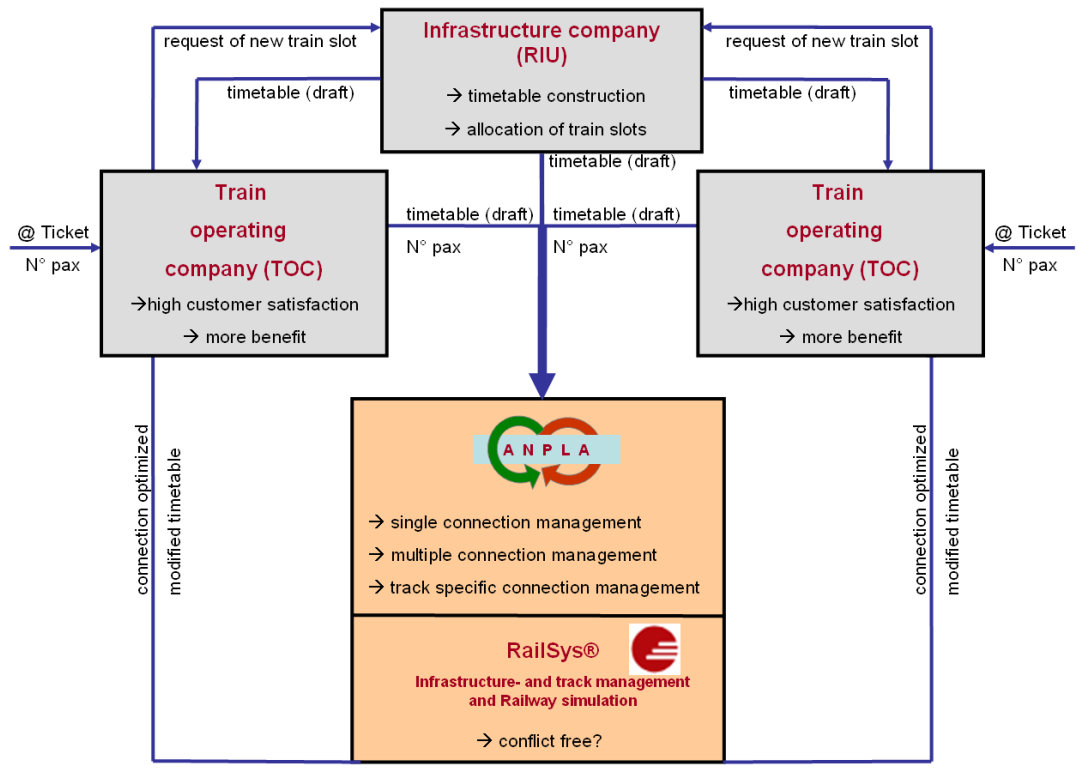

Figure 7: Timetable management workflow and application of ANPLA. 
simulation, ANPLA will be integrated in this process to perform the planning of various TOC driven passenger orientated strategies.

For this task, the TOC should submit their strategies or parts of their strategy regarding the passenger connection in stations to the RIU. These additional requests would enable the RIU to consider the TOC demands in a further dimension to construct timetables in a much better quality.

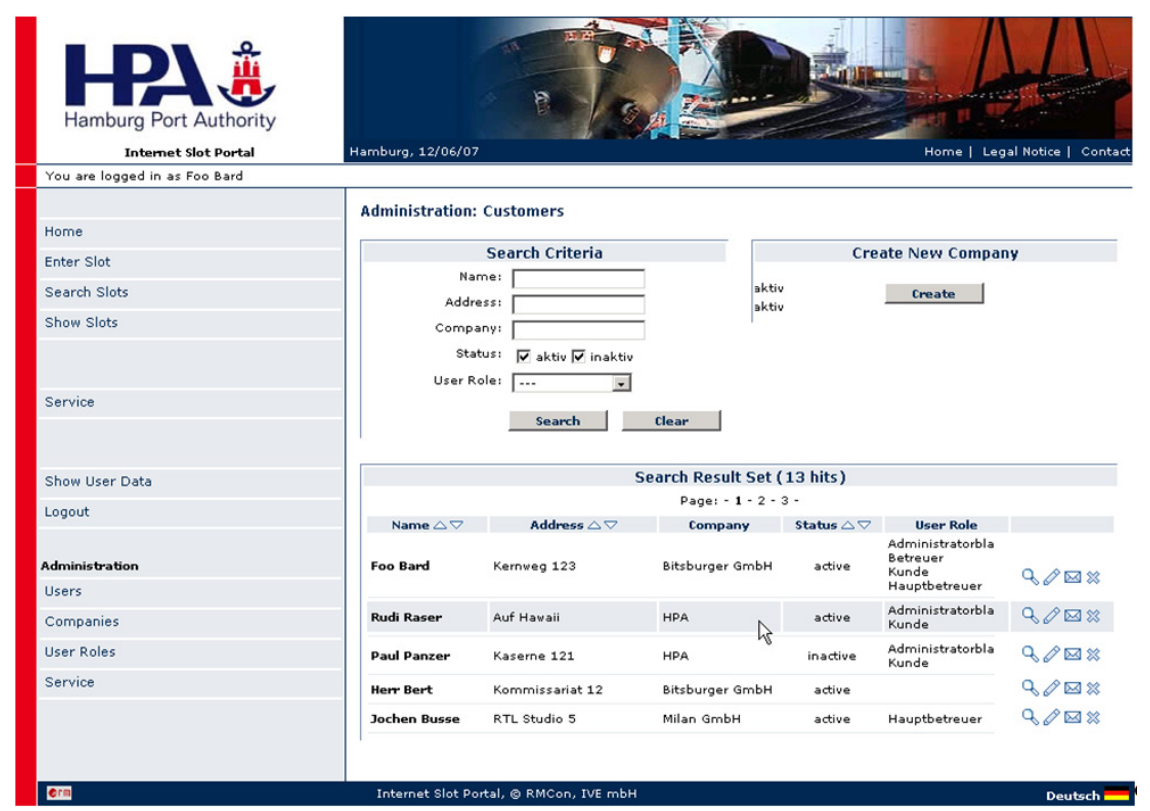

Figure 8: Example of the web interface of RailSys-CRM.

\section{Results and conclusions}

The overall result is the construction of a timetable with optimized total travel times and dwell times at connection stations in correlation of the service interval and the number of concerned passengers. Another result is a passenger concerned orientated new definition of waiting time rules. The potentials for implementation and the impacts for the railway business are for the timetable construction for train operating companies and for an improvement of the service quality of the transport offer by reducing the total travel time.

The new method of a passenger orientated connection management during the planning process

- reduces the transfer time,

- arises the proportion of direct connections,

- minimizes the travel time of all concerned passengers and

- integration into complex timetable planning environments. 


\section{References}

[1] Ahuja, R., Magnati, T., Orlin, J. "Network Flows", Prentice Hall, Upper Saddle River, New Jersey, 1993

[2] Radtke, A. "Software tools to model the railway operation", Habilitation, at the Institute of Transport Railway Construction and Operation, Leibniz University of Hanover, 2005

[3] Schumacher, A. "A hybrid method for vehicle allocation in rail traffic", Dissertation at the Institute of Transport Railway Construction and Operation, Leibniz University of Hanover, 2004

[4] Radtke, A.: "Timetable management and operational simulation: methodology and perspectives", presentation of COMPRAIL 2006, Prag Czech Republic, (2006), proceedings page 579 - 589

[5] RailSys Enterprise and RailSys CRM: www.rmcon.de

[6] Dispo: www.ivembh.de

[7] Klemenz, M. "Method of an optimized passenger orientated connection management during the planning process", Dissertation at the Institute of Transport Railway Construction and Operation, Leibniz University of Hanover, be published 2008 